\title{
Proton-pump Inhibitor-induced Severe Hypomagnesemia and Hypocalcemia are Clinically Masked by Thiazide Diuretic
}

\author{
Atsuko Uehara ${ }^{1}$, Yohei Kita $^{2}$, Hirofumi Sumi $^{3}$ and Yugo Shibagaki ${ }^{1}$
}

\begin{abstract}
:
Hypomagnesemia, a side effect of proton-pump inhibitors (PPIs), can be asymptomatic. The presence of hypocalcemia or hypokalemia is indicative of hypomagnesemia; however, the concomitant use of PPIs and thiazide may mask hypocalcemia. A 79-year-old woman with a history of chronic heart failure and chronic kidney disease developed symptomatic hypocalcemia and hypomagnesemia. Five weeks earlier, she had developed thiazide-induced hyponatremia, so thiazide had been discontinued. Reviewing the patient's charts revealed that three discontinued thiazide administrations in the clinical course had unmasked hypocalcemia. Our case demonstrates that thiazide-induced hypercalcemia can be so prominent as to mask PPI-induced hypocalcemia and hypomagnesemia.
\end{abstract}

Key words: hypocalcemia, hypomagnesemia, proton-pump inhibitor, thiazide-induced hypercalcemia

(Intern Med 58: 2201-2205, 2019)

(DOI: 10.2169/internalmedicine.2608-18)

\section{Introduction}

Hypomagnesemia is a common electrolyte disturbance that occurs in up to $12 \%$ of hospitalized patients and up to $60 \%$ to $65 \%$ of patients in the intensive-care unit (1). However, most cases of hypomagnesemia are asymptomatic and thus may be overlooked without routine checks. The key indicators of hypomagnesemia are other electrolyte disturbances triggered by hypomagnesemia, such as hypocalcemia and hypokalemia, which can often be symptomatic or be detected by reviewing laboratory test results (2). However, hypocalcemia and hypokalemia can also be asymptomatic or unnoticed when thiazide diuretics, vitamin $\mathrm{D}$, and reninangiotensin blockers are co-administered, which can make the diagnosis of hypomagnesemia difficult (3).

Given the frequency of hypomagnesemia and the fact that not only hypomagnesemia but also simultaneous hypocalcemia and hypokalemia can lead to fatal arrhythmia especially for patients with heart disease, it is crucial for clinicians to notice these electrolyte disturbances. We herein report a patient with proton-pump inhibitor (PPI)-induced chronic hypomagnesemia who presented with acute progressive and symptomatic hypocalcemia just after thiazide withdrawal.

\section{Case Report}

A 79-year-old woman with a medical history of ischemic heart disease, chronic heart failure, and chronic kidney disease [stage G4 or estimated glomerular filtration rate (GFR) of approximately $20 \mathrm{~mL} / \mathrm{min} / 1.73 \mathrm{~m}^{2}$ ] who was receiving thiazide diuretics for frequently exacerbated heart failure was admitted to our hospital because of persistent vomiting and hand tremors. Five weeks earlier, she had developed thiazide-induced hyponatremia, and thiazide treatment had been discontinued. Two weeks before her admission, she had experienced hand tremors. Two days before her admission, she experienced nausea and vomiting.

On an examination, resting hand tremors were observed, and Trousseau's sign was positive. There were no other neu-

${ }^{1}$ Division of Nephrology and Hypertension, Department of Internal Medicine, St. Marianna University School of Medicine, Japan, ${ }^{2}$ Department of Nephrology and Hypertension, St. Marianna University School of Medicine Yokohama City Seibu Hospital, Japan and ${ }^{3}$ Department of Nephrology and Hypertension, Kawasaki Municipal Tama Hospital, Japan

Received: December 31, 2018; Accepted: February 11, 2019; Advance Publication by J-STAGE: April 17, 2019

Correspondence to Dr. Atsuko Uehara, atsuko_mori@hotmail.com 
Table. Initial Laboratory Results.

\begin{tabular}{lcc}
\hline \multicolumn{1}{c}{ Parameter } & Level & Reference range \\
\hline Hemoglobin, g/dL & 10.9 & $11.0-14.0$ \\
Blood urea nitrogen, mg/dL & 20.8 & $8.0-22.0$ \\
Scr, mg/dL & 1.88 & $0.6-1.0$ \\
eGFR, mL/min/1.73 m² & 20.5 & $90-120$ \\
Serum albumin, g/dL & 3.5 & $4.1-5.1$ \\
Serum sodium, mEq/L & 145 & $135-140$ \\
Serum potassium, mEq/L & 3.8 & $3.5-5.1$ \\
Serum chloride, mEq/L & 106 & $97-109$ \\
Serum calcium, mg/dL & 5.3 & $8.8-10.3$ \\
Serum ionized calcium, mEq/L & 1.32 & $2.0-2.7$ \\
Serum phosphorus, mg/dL & 4.6 & $2.5-4.5$ \\
Serum magnesium, mEq/L & 0.3 & $1.4-2.2$ \\
Intact PTH, pg/mL & 111 & $10-65$ \\
25-hydroxyvitamin D, ng/mL & 7.4 & $20-50$ \\
1,25-dihydroxyvitamin D, pg/mL & 36 & $20-60$ \\
TRACP5b, mU/dL & 614 & $170-590$ \\
BALP, $\mu$ g/L & 20.6 & $3.7-20.9$ \\
Urine magnesium, mEq/L & 0.5 & NA \\
FEMg, \% & 8 & NA \\
Urine magnesium/creatinine ratio, mEq/g Cr & 1.0 & NA \\
FECa, \% & 1.4 & NA \\
Calcium/creatinine ratio, mg/g Cr & 21 & $<140$ \\
TmP/GFR, mg/dL & 5.7 & $2.3-4.3$ \\
Urine potassium/creatinine ratio, mEq/g Cr & 28.9 & NA \\
\hline Cr cing, BALP, bo aka/ne phosphtase, & & \\
\hline
\end{tabular}

Cr: creatinine, BALP: bone alkaline phosphatase, eGFR: estimated glomerular filtration rate, FECa: fractional excretion of calcium, FEMg: fractional excretion of magnesium, NA: not available, PTH: parathyroid hormone, Scr: serum creatinine, TRACP5b: tartrate-resistant acid phosphatase-5b, TmP/GFR: ratio of tubular maximum reabsorption rate of phosphate to glomerular filtration rate

rological findings. Her blood pressure was 107/68 $\mathrm{mmHg}$, heart rate was 84 beats/min, and respiratory rate was 23 breaths/min. Her body weight was $60.8 \mathrm{~kg}$, having decreased by $1.6 \mathrm{~kg}$ within the month that thiazide treatment was discontinued.

Initial laboratory tests showed hypocalcemia (albumincorrected serum calcium, $5.7 \mathrm{mg} / \mathrm{dL})$, hypomagnesemia $(0.3$ $\mathrm{mEq} / \mathrm{L})$, and normokalemia $(3.8 \mathrm{mEq} / \mathrm{L})$. Since her serum albumin concentration was $<4.0 \mathrm{~g} / \mathrm{dL}$, the albumin-corrected serum calcium concentration was calculated as total calcium $(\mathrm{mg} / \mathrm{dL})+0.8 \times[4.0$-serum albumin $(\mathrm{g} / \mathrm{dL})]$. The patient's laboratory results are shown in Table.

Electrocardiography showed a prolonged QT interval with a corrected QT interval of 0.61 seconds. She had been treated with furosemide for 7 years, PPIs for 3 years (esomeprazole in the first year and lansoprazole in the subsequent years), and trichlormethiazide for 2 years.

Subsequent investigations revealed a plasma intact parathyroid hormone (PTH) level of $111 \mathrm{pg} / \mathrm{mL}$ (reference range, 10-65), 25-hydroxyvitamin $\mathrm{D}$ level of $7.4 \mathrm{ng} / \mathrm{mL}$ (reference range, 20-50), and 1,25-dihydroxyvitamin $\mathrm{D}$ level of $36 \mathrm{pg} / \mathrm{mL}$ (reference range, 20-60). Her urinary magnesium concentration was $0.5 \mathrm{mEq} / \mathrm{L}$, and urinary magnesium/creatinine ratio was $1.0 \mathrm{mEq} / \mathrm{g}$ creatinine. Her urinary potas- sium/creatinine ratio was $28.9 \mathrm{mEq} / \mathrm{g}$ creatinine. The renal threshold of phosphate reabsorption standardized to glomerular filtration rate (TmP/GFR) was $5.7 \mathrm{mg} / \mathrm{dL}$. The level of tartrate-resistant acid phosphatase 5b (TRACP5b), a bone resorptive marker, was $614 \mathrm{mU} / \mathrm{dL}$ (reference range, 170-590), while that of bone alkaline phosphatase (BALP), a bone formation marker, was $20.6 \mu \mathrm{g} / \mathrm{L}$ (reference range, 3.7-20.9, Table).

Her fractional excretion of magnesium (FEMg) was as high as $8 \%$, which suggested that her kidneys were inappropriately excreting magnesium despite her hypomagnesemia. However, we interpreted this result with caution (2) because in the setting of chronic kidney disease with a low GFR, the reference range of FEMg increases, so other indices, such as the total 24-hour urinary excretion of magnesium, urinary magnesium concentration, and urinary magnesium/creatinine ratio, are more accurate than FEMg for evaluating the urinary magnesium excretion (4). Although our patient had been treated with loop diuretics, which can cause hypomagnesemia, her urinary magnesium level and urinary magnesium/creatinine ratio were low, suggesting that the hypomagnesemia was caused by extrarenal magnesium loss or transcellular magnesium shift. However, our patient did not have a condition like refeeding syndrome, in which magnesium 
shifts from the extracellular fluid into the intracellular fluid. Since this patient had been treated with PPIs and lacked any other potential causes of gastrointestinal magnesium loss, such as diarrhea, and given that PPIs are known to induce gastrointestinal magnesium loss and lead to hypomagnesemia $(5,6)$, PPI-induced hypomagnesemia was strongly suspected.

We suspected that her hypocalcemia was partly due to vitamin D deficiency, as suggested by her low 25hydroxyvitamin D level and relatively low 1,25dihydroxyvitamin D level for cases of profound hypocalcemia. However, hypomagnesemia is known to cause hypocalcemia due to decreased PTH secretion and increased PTH resistance (7-9). Her PTH level was slightly higher than the reference level because of her low GFR but was relatively low for her profound hypocalcemia, suggesting that she had reduced PTH secretion. In addition, her 1,25dihydroxyvitamin D level was relatively low for her PTH level, suggesting increased PTH resistance or a low GFR. Thiazide diuretics are known to increase the serum calcium level, which masks hypocalcemia; we therefore concluded that the thiazide withdrawal one month before her admission in this case triggered acute progressive and symptomatic hypocalcemia (10).

Although hypokalemia was not noted, her urinary potassium excretion rate was relatively high. Given that her serum potassium level was only slightly above the lower limit of normal, we assumed there was relative potassium overexcretion due to hypomagnesemia. However, her renal insufficiency limited urinary potassium excretion and might have masked the hypokalemia.

Given these findings, PPI use was discontinued, and 80 $\mathrm{mEq}$ of magnesium and $314 \mathrm{mg}$ of calcium were administered to the patient intravenously over 24 hours, along with $0.5 \mu \mathrm{g}$ of alfacalcidol. The following day, her albumincorrected serum calcium and magnesium levels increased to $6.5 \mathrm{mg} / \mathrm{dL}$ and $2.0 \mathrm{mEq} / \mathrm{L}$, respectively, and her symptoms subsided; we therefore stopped administering magnesium and calcium supplements. After PPI withdrawal, her serum magnesium level normalized; hypomagnesemia did not recur, and her urinary magnesium excretion increased. Eleven days later, the serum magnesium and calcium levels were normal, and the intact PTH level increased to $160 \mathrm{pg} / \mathrm{mL}$, suggesting that PTH secretion had been suppressed by the hypomagnesemia. The patient was discharged from the hospital on day 17.

\section{Discussion}

We encountered a case of PPI-induced hypomagnesemia that had been asymptomatic and overlooked for years because thiazide diuretics masked the hypocalcemia, which is indicative of hypomagnesemia. This report emphasizes the fact that thiazide-induced hypercalcemia is occasionally so prominent it masks hypocalcemia and hypomagnesemia induced by PPIs. Upon reviewing the patient's medical charts, we noted that the patient's serum magnesium levels had been checked only twice prior to her admission to our hospital, and we found that the patient had experienced hypomagnesemia 2 years earlier. Four years ago, her serum magnesium level had been $1.8 \mathrm{mEq} / \mathrm{L}$, and 2 years ago, it had been $0.3 \mathrm{mEq} / \mathrm{L}$, but the hypomagnesemia went unnoticed and untreated. The fact that PPI was started 3 years ago supports our hypothesis that hypomagnesemia was induced by the PPI therapy. A loop diuretic had been started 7 years earlier, and her serum magnesium level had been subsequently normalized within 3 years; this finding excludes the involvement of loop diuretics in the pathogenesis of hypomagnesemia.

A case series of PPI-induced hypomagnesemia showed that hypomagnesemia was caused by gastrointestinal magnesium loss, as revealed by the patients' very low urinary magnesium level and very high fecal magnesium level (6). The small and large intestines are responsible for magnesium absorption via passive and active transport. Transient receptor potential melastatin (TRPM) 6 plays a vital role in actively reabsorbing magnesium, especially with decreased magnesium levels (11). When PPIs are administered, the $\mathrm{pH}$ of the intestinal lumen increases, and the activity of $\mathrm{pH}$ dependent TRPM6 channels decreases, hindering magnesium reabsorption.

In the current case, the patient's symptomatic hypocalcemia was a key indicator of the diagnosis of hypomagnesemia. Hypomagnesemia suppresses PTH secretion by activating calcium-sensing receptors $(7,8)$. In addition, hypomagnesemia increases PTH resistance by inhibiting PTH and receptor binding, which suppresses PTH intracellular signaling (9). Generally, PTH resistance is judged by its end-organ responsiveness, namely vitamin D activity, renal phosphate excretion, and bone metabolism (12). In the current case, the fact that the patient's 1,25-dihydroxyvitamin D level was relatively low and her TmP/GFR was high suggests the existence of PTH resistance in the kidney (13). However, her TRACP5b level was high, and her BALP level was just below the upper limit of normal, which means that the bone responsiveness to PTH was sustained. This high bone turnover was considered to have been caused by a slightly elevated PTH level and postmenopausal osteoporosis $(14,15)$. Thus, decreased intestinal calcium reabsorption, not decreased calcium release from the bone, was related to her hypocalcemia. Significant and progressive hypocalcemia was seen in our patient. Her albumin-corrected serum calcium decreased from 8.6 to $5.8 \mathrm{mg} / \mathrm{dL}$ after thiazide withdrawal. Although her calcium level was low and she was experiencing volume depletion, based on her body weight loss, her fractional excretion of calcium was relatively high. We therefore considered that this significant decrease in calcium level was due at least in part to the calciuretic effect of furosemide in addition to thiazide withdrawal. It has also been reported that magnesium and vitamin $\mathrm{D}$ interact in a coordinated manner in the body. The metabolism of vitamin $\mathrm{D}$ by hepatic 25-hydroxylation and renal $1 \alpha$-hydroxylation is a 


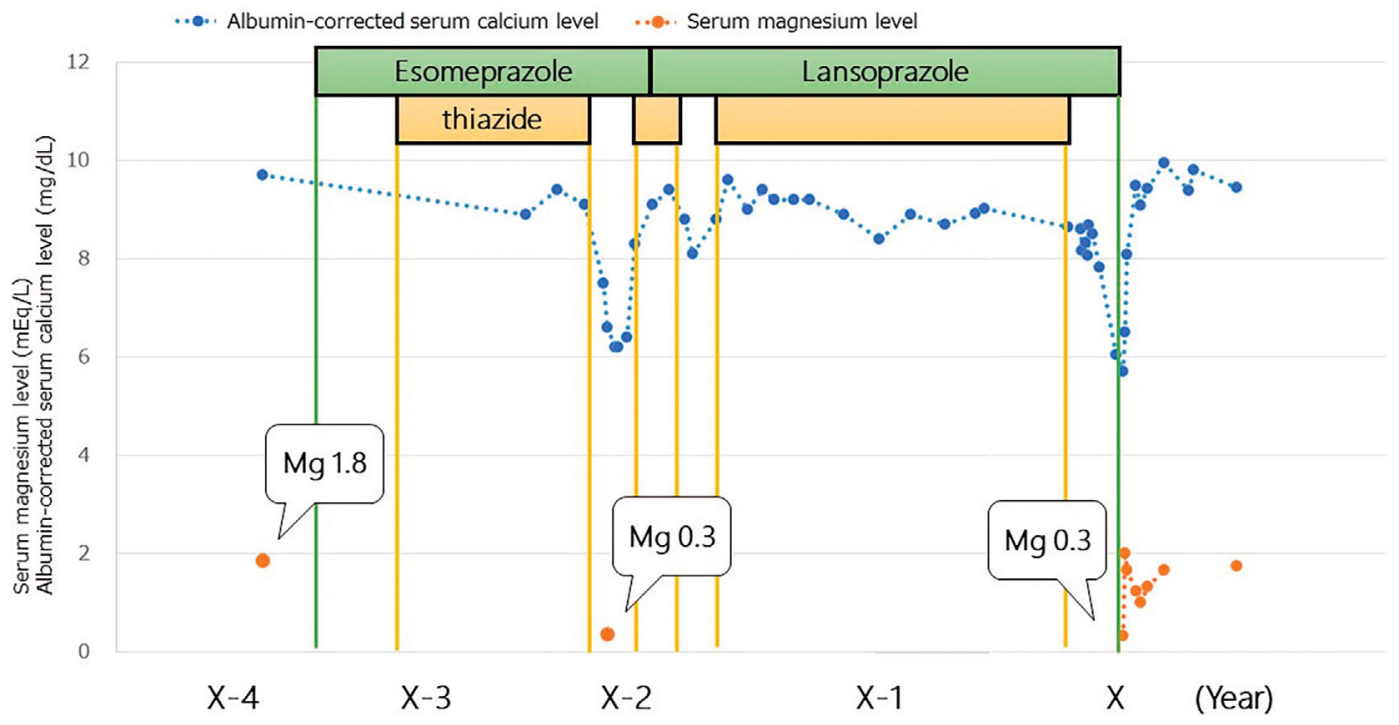

Figure. Serum calcium and magnesium levels following the start of PPI therapy. Periods of PPI and thiazide diuretic use are shown. The patient took furosemide $20 \mathrm{mg}$ throughout the entire course. The patient experienced hypocalcemia three times, all instances of which occurred after the withdrawal of thiazide diuretics. Mg: magnesium, PPI: proton-pump inhibitor

magnesium-dependent process. In addition, 1,25dihydroxyvitamin $\mathrm{D}$ itself stimulates intestinal magnesium absorption (16). Therefore, in our case, the low vitamin D level was both a result of hypomagnesemia and a cause of hypomagnesemia and hypocalcemia.

It is worth clarifying why symptomatic hypocalcemia did not occur during the four-year course of PPI therapy. A review of the patient's chart revealed that she had experienced hypocalcemia twice, both instances of which occurred after thiazide withdrawal, and her lowest albumin-corrected serum calcium levels were 6.2 and $8.1 \mathrm{mg} / \mathrm{dL}$, consecutively (Figure). In addition, at that time, she had symptoms of hypocalcemia and complained of nausea; hypocalcemia was subsequently detected. However, only oral calcium supplements without vitamin D were administered, and the hypocalcemia subsided after thiazides were resumed for the management of congestive heart failure, after which calcium supplementation was withdrawn. Hypomagnesemia and hypocalcemia had been diagnosed 3 years ago, but no intervention was initiated to treat the hypomagnesemia. We hypothesized that symptomatic and profound hypocalcemia had not developed over the previous 2 years because of the concomitant use of thiazide diuretics that masked the hypocalcemia by increasing renal calcium reabsorption; thus, thiazide withdrawal 5 weeks before her presentation had triggered progressive hypocalcemia (17). Although it is commonly held that the renal response to thiazides may be suboptimal with an eGFR of $20 \mathrm{~mL} / \mathrm{min} / 1.73 \mathrm{~m}^{2}$, as in our patient, many studies of thiazides in advanced CKD have reported some degree of efficacy, whether for hypertension or diuresis (18). In addition, our patient had been taking a loop diuretic for several years, which induced hypertrophy of the distal convoluted tubule and made this site more sensitive to thiazide.
Our findings indicate that even severe hypomagnesemia might be overlooked when thiazide is used concomitantly. Although primary hyperparathyroidism unmasked by thiazide-induced hypercalcemia was described in a previous report, to our knowledge, this is the first report to describe hypocalcemia unmasked by thiazide withdrawal (19). It is important to note that thiazide treatment can mask hypocalcemia, making it easy to overlook hypomagnesemia. It is noteworthy that hypomagnesemia and subsequent hypokalemia and hypocalcemia can cause arrhythmia (20). Importantly, even mild hypomagnesemia is a risk factor for left ventricular hypertrophy and cardiovascular events, which lead to increased mortality (21).

Although the development of hypomagnesemia by PPI is not rare, the prevention of hypocalcemia by thiazide diuretics is unique and may benefit readers as a warning case; we therefore strongly suggest that clinicians should routinely assess the serum magnesium and calcium levels during PPI and thiazide therapy.

The authors state that they have no Conflict of Interest (COI).

\section{References}

1. Agus ZS. Hypomagnesemia. J Am Soc Nephrol 10: 1616-1622, 1999.

2. Dimke H, Monnens L, Hoenderop JG, Bindels RJ. Evaluation of hypomagnesemia: lessons from disorders of tubular transport. Am J Kidney Dis 62: 377-383, 2013.

3. Kim MJ, Kim JH, Kim IY, Lee DW, Lee SB. Reninoma masked by the use of an angiotensin receptor blocker. Iran J Kidney Dis 10: 413-415, 2016.

4. Cunningham J, Rodríguez M, Messa P. Magnesium in chronic kidney disease Stages 3 and 4 and in dialysis patients. Clin Kidney J 5 (Suppl): i39-i51, 2012. 
5. Cundy T, Dissanayake A. Severe hypomagnesaemia in long-term users of proton-pump inhibitors. Clin Endocrinol (Oxf) 69: 338341, 2008.

6. Hoorn EJ, van der Hoek J, de Man RA, Kuipers EJ, Bolwerk C, Zietse R. A case series of proton pump inhibitor-induced hypomagnesemia. Am J Kidney Dis 56: 112-116, 2010.

7. Quitterer U, Hoffmann M, Freichel M, Lohse MJ. Paradoxical block of parathormone secretion is mediated by increased activity of G alpha subunits. J Biol Chem 276: 6763-6769, 2001.

8. Vetter T, Lohse MJ. Magnesium and the parathyroid. Curr Opin Nephrol Hypertens 11: 403-410, 2002.

9. Freitag JJ, Martin KJ, Conrades MB, et al. Evidence for skeletal resistance to parathyroid hormone in magnesium deficiency. Studies in isolated perfused bone. J Clin Invest 64: 1238-1244, 1979.

10. Griebeler ML, Kearns AE, Ryu E, et al. Thiazide-associated hypercalcemia: incidence and association with primary hyperparathyroidism over two decades. J Clin Endocrinol Metab 101: 11661173,2016

11. William JH, Danziger J. Proton-pump inhibitor-induced hypomagnesemia: current research and proposed mechanisms. World $\mathrm{J}$ Nephrol 5: 152-157, 2016.

12. Yamamoto M, Yamaguchi $T$, Yamauchi M, Yano S, Sugimoto $T$. Acute-onset hypomagnesemia-induced hypocalcemia caused by the refractoriness of bones and renal tubules to parathyroid hormone. J Bone Miner Metab 29: 752-755, 2011

13. Bacchetta J, Salusky IB. Evaluation of hypophosphatemia: lessons from patients with genetic disorders. Am J Kidney Dis 59: 152-
159, 2012.

14. Morris HA, Eastell R, Jorgensen NR, et al. Clinical usefulness of bone turnover marker concentrations in osteoporosis. Clin Chim Acta 467: 34-41, 2017.

15. Eastell R, Szulc P. Use of bone turnover markers in postmenopausal osteoporosis. Lancet Diabetes Endocrinol 5: 908-923, 2017.

16. Uwitonze AM, Razzaque MS. Role of magnesium in vitamin D activation and function. J Am Osteopath Assoc 118: 181-189, 2018.

17. Reilly RF, Huang CL. The mechanism of hypocalciuria with $\mathrm{NaCl}$ cotransporter inhibition. Nat Rev Nephrol 7: 669-674, 2011.

18. Sinha AD, Agarwal R. Thiazides in advanced chronic kidney disease: time for a randomized controlled trial. Curr Opin Cardiol 30: 366-372, 2015.

19. Wermers RA, Kearns AE, Jenkins GD, Melton LJ 3rd. Incidence and clinical spectrum of thiazide-associated hypercalcemia. Am J Med 120: 911.e9-911.e15, 2007.

20. El-Charabaty E, Saifan C, Abdallah M, et al. Effects of proton pump inhibitors and electrolyte disturbances on arrhythmias. Int $\mathbf{J}$ Gen Med 6: 515-518, 2013.

21. Reffelmann $\mathrm{T}$, Ittermann $\mathrm{T}$, Dörr M, et al. Low serum magnesium concentrations predict cardiovascular and all-cause mortality. Atherosclerosis 219: 280-284, 2011.

The Internal Medicine is an Open Access journal distributed under the Creative Commons Attribution-NonCommercial-NoDerivatives 4.0 International License. To view the details of this license, please visit (https://creativecommons.org/licenses/ by-nc-nd/4.0/).

(C) 2019 The Japanese Society of Internal Medicine

Intern Med 58: 2201-2205, 2019 